\title{
Millimeter Wave Hybrid Beamforming with DFT-MUB Aided Precoder Codebook Design
}

\author{
K. Satyanarayana*, Mohammed El-Hajjar*, Ping-Heng Kuo ${ }^{\dagger}$, Alain Mourad ${ }^{\dagger}$, Lajos Hanzo* \\ ${ }^{*}$ Department of Electronics and Computer Science, University of Southampton, UK. \\ ${ }^{\dagger}$ InterDigital, Europe, London, UK. \\ Email: \{ks1r15, meh, lh\}@ecs.soton.ac.uk, \{ping-heng.kuo, alain.mourad $\} @$ InterDigital.com
}

\begin{abstract}
The millimeter wave (mmWave) frequency band offers substantial hitherto unused spectral resources for future wireless communication systems in order to meet the increasing capacity demand. However, mmWave frequencies suffer from high propagation losses, which may be mitigated by directional beamforming in addition to baseband precoding. This is usually referred to as hybrid beamforming. In this paper, we investigate the so-called discrete Fourier transform-mutually unbiased bases (DFT-MUB) aided codebook design conceived for limitedfeedback mmWave systems, where the MUB aided codebook is applied in the baseband, while the DFT codebook is invoked for RF analog phase shifters. We demonstrate that our DFTMUB codebook design performs similarly to the optimal digital precoding matrix, where the precoder is selected as the right singular matrix of the channel. However, our solution significantly reduces the search complexity in the baseband, while performing within $2.5 \mathrm{~dB}$ from the optimal digital precoder.
\end{abstract}

\section{INTRODUCTION}

Recent studies show a dearth of spectral resources to meet the increasing data rate demands of mobile users in the microwave band [1]. Hence next generation systems aim for additionally harnessing mmWave frequencies [2] as a benefit of the abundant availability of bandwidth. However, mmWave frequencies suffer from a high pathloss because of the attenuation due to water vapour, oxygen absorption and foliage. As a result, the signal-to-noise (SNR) ratio in mmWave communications would typically be low at the receiver [2]. To compensate the propagation losses, directional beamforming is considered to be a promising solution [3]. Furthermore, since the $\lambda / 2$ spacing between the antennas would be on the order of millimeters at mmWave frequencies, large number of antennas can be accommodated in a compact space for achieving high beamforming gains. Employing digital beamforming using a large number of antennas, where both the amplitude and phase are controlled digitally using ADCs/DACs, would result in a high cost and hardware complexity [4]. On the other hand, employing analog beamforming, where the antenna array is connected to an RF chain through amplifiers and controllable analog phase shifters, reduces the hardware complexity significantly compared to the digital beamforming. However, in analog beamforming, the angular resolution is typically inaccurate [2]. Hence hybrid beamforming combining analog and digital beamforming for mmWave systems was conceived in [5], where the ensuing digital processing

The financial support of InterDigital, UK and of Beam-Me-Up project is gratefully acknowledged. is capable of correcting the analog imperfections. In hybrid solutions, analog beamforming is carried out at the RF front end, where the digitally precoded signals are phase-shifted and then fed to the transmit antennas. Similarly, at the receiver RF front end the received signals are phase-shifted and then fed to the digital combiner operating in the baseband. Two different hybrid beamforming designs have been reported in [6], namely a so-called full-array-connected design and, subarray-connected design [6]. In the full-array-connected design the digital precoded signals are phase-shifted and then fed to all the transmit antennas. By contrast, in the sub-arrayconnected design, after phase shifting the digitally precoded signals are fed only to a subset of the antennas. Hence, the sub-array-connected implementation is more energy efficient than the full-array-connected design [6].

In frequency-division duplex (FDD) systems, the receiver has to feed back the channel estimation to the transmitter for the transmit precoder (TPC) to compensate the channel-effects about to be encountered. However, feeding back the channel coefficients to the transmitter would result in large overhead. An appealing practical solution for feeding back the channel information to the transmitter is based on a carefully designed TPC quantized weight codebook, which is known both to the transmitter and the receiver. Explicitly, based upon the channel impulse response (CIR) estimate the receiver selects the best TPC matrix from its codebook, and reports the selected index to the transmitter over the feedback channel. This technique is referred to as limited-feedback design.

Song et al. [7] proposed a codebook design for mmWave systems using the algorithm referred to as orthogonal matching pursuit (OMP). As a futher solution, Rajashekar and Hanzo [8] designed a codebook design using vector quantization conceived for finite modulation alphabets. In [9], Alkhateeb and Heath proposed RF codebook designs for limited feedback mmWave systems. By exploiting the channels' reciprocity, a heuristic codebook design was advocated by Eltayeb et al. [10]. The efficiency of a practical communication system critically hinges on its performance vs complexity tradeoff. Moreover, the aforementioned designs mmWave system designs impose a high complexity. The performance of a practical codebook design depends on the choice of the codebook entries, as detailed by Clerckx et al. in [11]. Against this background, in this contribution, we conceive a lowcomplexity hybrid beamforming codebook design. 
More explicitly, we design a codebook for digital beamforming based on mutually unbiased bases (MUBs) combined with discrete Fourier transform (DFT) based analog RF beamforming for a limited-feedback aided mmWave MIMO system. The MUB assisted codebook imposes lower search complexity than the existing digital beamforming designs without compromising the attainable performance. A MUB assisted codebook was designed in [12], [13] for a sub-6 GHz scenario. By contrast, we design a codebook based on MUBs combined with DFT aided analog RF beamforming for a limited-feedback mmWave MIMO system. The rationale behind employing the DFT for analog RF beamforming is its high efficiency in spatially correlated channels [14]. Our simulation results demonstrate that despite of its low complexity, the proposed MUB-DFT codebook design performs close to the equivalent benchmarker applying digital precoding and relying on the simplifying assumption of having perfect channel state information (CSI).

The rest of the paper is organized as follows. In Sec. II, we discuss our system model as well as the design of the transmitter and receiver in addition to the mmWave channel model. In Sec. III, we first discuss the DFT aided codebook design conceived for analog RF beamforming. Later we focus our attention on MUB assisted codebook design for the baseband. Our simulation results and conclusions are presented in Sec. IV and Sec. V, respectively.

Notations: We use upper case boldface, $\mathbf{A}$, for matrices and lower case boldface, a, for vectors. We use $<.>, T, H, \otimes$ for the inner product, transpose, Hermitian transpose and elementwise multiplication, respectively. Finally, we use $\mathcal{C N}, \mathcal{U}$, and i.i.d. to denote complex-valued normal distribution, uniform distribution, and independent and identical distribution, respectively.

\section{System ModeL}

Consider a single-user mmWave MIMO system shown in Fig. 1, where the transmitter is equipped with $N_{t}$ antennas and the receiver with $N_{r}$ antennas. Then the received signal vector after RF and baseband combining is given by:

$$
\mathbf{y}=\sqrt{P} \mathbf{W}_{\mathrm{BB}}^{H} \mathbf{W}_{\mathrm{RF}}^{H} \mathbf{H F}_{\mathrm{RF}} \mathbf{F}_{\mathrm{BB}} \mathbf{s}+\mathbf{W}_{\mathrm{BB}}^{H} \mathbf{W}_{\mathrm{RF}}^{H} \mathbf{n},
$$

where $\mathbf{H}$ is the statistical channel model expressed as [15]:

$$
\mathbf{H}=\sqrt{\frac{N_{r} N_{t}}{N_{c} N_{\text {ray }}}} \sum_{n_{c}=1}^{N_{c}} \sum_{n_{\text {ray }}=1}^{N_{\text {ray }}} \alpha_{n_{c}}^{n_{\text {ray }}} \mathbf{a}_{r}\left(\phi_{n_{c}}^{n_{\text {ray }}}\right) \mathbf{a}_{t}^{T}\left(\phi_{n_{c}}^{n_{\text {ray }}}\right),
$$

and the transmitter is equipped with $N_{t}^{\mathrm{RF}}$ chains and the receiver with $N_{r}^{\mathrm{RF}}$ chains, where $\mathbf{F}_{\mathrm{RF}}$ is the $\mathrm{RF}$ beamformer matrix of size $N_{t} \times N_{t}^{\mathrm{RF}}, \mathbf{F}_{\mathrm{BB}}$ is the baseband TPC matrix of size $N_{t}^{\mathrm{RF}} \times N_{s}, \mathbf{W}_{\mathrm{RF}}^{H}$ is the RF combiner matrix of size $N_{r}^{\mathrm{RF}} \times N_{r}, \mathbf{W}_{\mathrm{BB}}^{H}$ is the baseband combiner of size $N_{s} \times N_{r}^{\mathrm{RF}}, \mathbf{y}$ is the $N_{s} \times 1$ received vector, $\mathbf{s}$ is the symbol vector of size $N_{s}$, where $N_{s}<N_{t}^{\mathrm{RF}}$ and $\mathbf{n}$ is the noise vector of i.i.d. $\mathcal{C N}\left(0, \sigma^{2}\right)$. Furthermore, $\mathbf{H}$ is the statistical channel matrix of size $N_{r} \times N_{t}$ so that $\mathbb{E}\left[\|\mathbf{H}\|_{F}^{2}\right]=N_{t} N_{r}$, while $\alpha_{n_{c}}^{n_{\text {ray }}} \sim \mathcal{C N}(0,1)$ is a complex-valued Gaussian random variable, whose amplitude and phase are Rayleigh and uniform distributed, respectively. For a uniform linear array (ULA) with $N_{r}$ and $N_{t}$ antenna elements the response vectors $\mathbf{a}_{r}$ and $\mathbf{a}_{t}$ are expressed as:

$$
\begin{aligned}
& \mathbf{a}_{r}\left(\phi_{r}\right)=\left[1 e^{j \frac{2 \pi}{\lambda} d \cos \left(\phi_{r}\right)} \ldots e^{j \frac{2 \pi}{\lambda}\left(N_{r}-1\right) d \cos \left(\phi_{r}\right)}\right]^{T}, \\
& \mathbf{a}_{t}\left(\phi_{t}\right)=\left[1 e^{j \frac{2 \pi}{\lambda} d \cos \left(\phi_{t}\right)} \ldots e^{j \frac{2 \pi}{\lambda}\left(N_{t}-1\right) d \cos \left(\phi_{t}\right)}\right]^{T} .
\end{aligned}
$$

Finally, $N_{c}$ and $N_{\text {ray }}$ are the number of CIR clusters and rays, respectively.

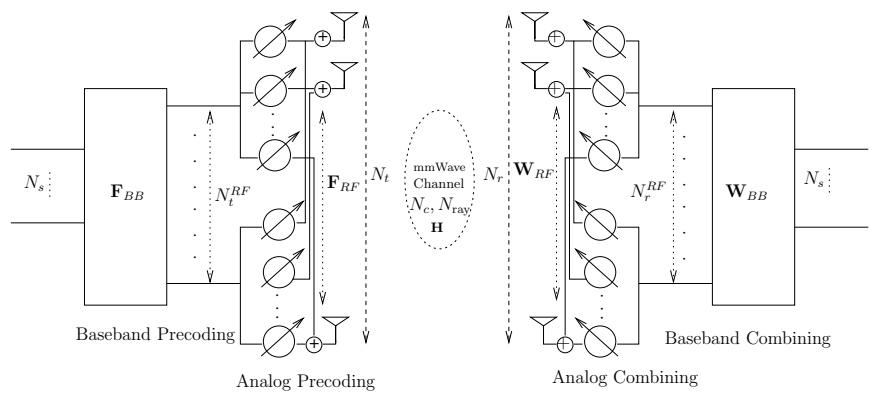

Fig. 1: Hybrid beamforming transceiver architecture.

In mmWave communication, $\mathbf{F}_{\mathrm{RF}}$ and $\mathbf{W}_{\mathrm{RF}}$ are used for analog beamforming, where the beams are steered in the direction of the desired user by phase shifters, while $\mathbf{F}_{\mathrm{BB}}$ and $\mathbf{W}_{\mathrm{BB}}$ are the baseband precoder and combiner weights used for mitigating the inter-stream interference. With the aid of this system model, the achievable rate in bits per second per channel use (bps/cu) is given by:

$$
\begin{aligned}
& C=\log _{2} \mid \operatorname{det}\left(\mathbf{I}_{N_{s}}+\right. \\
& \left.\frac{P}{N_{s}} \mathbf{R}_{n}^{-1} \mathbf{W}_{\mathrm{BB}}^{H} \mathbf{W}_{\mathrm{RF}}^{H} \mathbf{H F}_{\mathrm{RF}} \mathbf{F}_{\mathrm{BB}} \mathbf{F}_{\mathrm{BB}}^{H} \mathbf{F}_{\mathrm{RF}}^{H} \mathbf{H}^{H} \mathbf{W}_{\mathrm{RF}} \mathbf{W}_{\mathrm{BB}}\right) \mid,
\end{aligned}
$$

where $\mathbf{R}_{n}=\sigma^{2} \mathbf{W}_{\mathrm{BB}}^{H} \mathbf{W}_{\mathrm{RF}}^{H} \mathbf{W}_{\mathrm{RF}} \mathbf{W}_{\mathrm{BB}}$, and $P$ is the signal power.

\section{CODEBOOK Design}

Fig. 1 shows the transceiver design relying both on a TPC and on analog beamforming. In this design, before the streams are fed to the transmit antennas, they are precoded in the baseband using a linear TPC and then the precoded streams are fed to the RF beamformer, where the streams are phase shifted accordingly and finally fed to the transmit antennas.

In this section, we discuss the design of RF beamforming based on the DFT, where the antenna element weights are selected for steering the beam in the desired direction. Then, we describe the construction of the baseband TPC codebook relying on MUBs.

\section{A. RF Analog Beamformer Design Using DFT}

To derive the beamforming gains so as to compensate for the propagation losses due to water vapour, oxygen absorption and foliage, the antennas are placed at a separation of $\lambda / 2$. We consider the DFT based codebook for RF beamforming, because the codewords in the DFT based beamforming codebook match with the statistical distribution of the optimal beamforming weight vectors, which makes the DFT based codebook more efficient in spatially correlated channels [14]. Furthermore, it satisfies the constant modulus constraint imposed by the phase shifters to avoid any power imbalance for 
the system. Therefore, it is reasonable to employ a DFT based codebook for analog RF beamforming using phase shifters. On the other hand, for the baseband TPC matrix, we invoke a codebook design constructed from MUBs as a benefit of its efficiency and low complexity, which will be detailed later in this section.

The $N_{t}^{\mathrm{RF}}$ columns of the analog RF beamformer matrix $\mathbf{F}_{\mathrm{RF}}$ are selected from the $N_{t} \times N_{t}$ DFT matrix which exhibits maximum correlation with the columns of the right singular matrix of the channel $\mathbf{H}=\mathbf{U} \Sigma \mathbf{V}^{H}$, i.e.,

$$
\mathbf{F}_{\mathrm{RF}}(:, i)=\max _{i}<\mathbf{D F T}_{N_{t}}(:, i), \mathbf{V}>, 1 \leq i \leq N_{t}^{\mathrm{RF}},
$$

where $\mathbf{V}$ is the right singular matrix of the channel and DFT $_{N_{t}}(:, i)$ is the $i^{t h}$ column of the $N_{t} \times N_{t}$ DFT matrix. Similarly, the $N_{r}^{\mathrm{RF}}$ columns of $\mathbf{W}_{\mathrm{RF}}$ are selected from the $N_{r} \times N_{r}$ DFT matrix which exhibits maximum correlation with the columns of the left singular matrix of the channel $\mathbf{H}$, i.e.,

$$
\mathbf{W}_{\mathrm{RF}}(:, i)=\max _{i}<\mathbf{D F T}_{N_{r}}(:, i), \mathbf{U}>, 1 \leq i \leq N_{r}^{\mathrm{RF}},
$$

where $\mathbf{U}$ is the left singular matrix of the channel and $\mathbf{D F T}_{N_{t}}($ : , $i$ ) is the $i^{\text {th }}$ column of the $N_{r} \times N_{r}$ DFT matrix.

After applying the analog RF beamformer weights, the channel seen by the baseband TPC after RF beamforming is the effective channel given by:

$$
\mathbf{H}_{\mathrm{eff}}=\mathbf{W}_{\mathrm{RF}}^{H} \mathbf{H F}_{\mathrm{RF}} \text {. }
$$

We now design the baseband codebook using MUBs described in the next section.

\section{B. Baseband Codebook Design Using MUBs}

The theory of MUB and the associated codebook design are described in this section. Let $\mathbf{B}$ and $\mathbf{B}^{\prime}$ be a pair of orthonormal bases of an $N$-dimensional Hilbert space. Then, $\mathbf{B}$ and $\mathbf{B}^{\prime}$ are said to be mutually unbiased if and only if [16]:

$$
\left|<\mathbf{b}, \mathbf{b}^{\prime}>\right|^{2}=\frac{1}{N}, \forall \mathbf{b} \in \mathbf{B} \text { and } \mathbf{b}^{\prime} \in \mathbf{B}^{\prime},
$$

where $\mathbf{b}$ and $\mathbf{b}^{\prime}$ are vectors in $\mathbf{B}$ and $\mathbf{B}^{\prime}$, respectively. The codebook constructed from MUBs ensures having low distances between the codewords [12].

However, the number of MUBs for a given dimension is limited, which affects the size of the codebook. Furthermore, the number of antenna elements should be a power of a prime [16], then the number of MUBs available for $N_{t}^{\mathrm{RF}}$ dimension is $N_{t}^{\mathrm{RF}}+1$ [16]. Moreover, if the number of RF chains $N_{t}^{\mathrm{RF}}$ is a power of 2 , then the entries of the MUB matrix is observed to be composed of finite alphabets i.e., $\{1,-1, i,-i\}$. Additionally, if $\left\{\mathbf{F}_{1}, \ldots, \mathbf{F}_{N}\right\}$ are the codewords, then the minimum distance $d_{\min }$ between the codewords is given by:

$$
d_{\min }=\min _{\mathrm{k}, 1 ; k \neq l}\left\|\mathbf{F}_{k} \mathbf{F}_{k}^{H}-\mathbf{F}_{l} \mathbf{F}_{l}^{H}\right\|
$$

which is close to the theoretical bound [12].

Codebook construction: For a dimension of $N_{t}^{\mathrm{RF}}=2^{n}$, where $n$ is a natural number, we commence our discourse from a Hadamard matrix $\mathbf{A}$ of dimension $2^{n}$, which satisfies the property of a MUB. Using the Hadamard matrix A, we can construct another basis $\mathbf{B}$ by finding a column vector $\mathbf{v}$, so that all the elements in $\mathbf{A}^{H} \mathbf{v}$ have a magnitude equal to 1 . Then we obtain the basis $\mathbf{B}$ by element-wise multiplication of $\mathbf{v}$ with $\mathbf{A}$, i.e., if $\mathbf{A}=\left[\begin{array}{lll}\mathbf{a}_{1} & \mathbf{a}_{2} \ldots \mathbf{a}_{2^{n}}\end{array}\right]$ then $\mathbf{B}=\mathbf{v} \otimes \mathbf{A}_{2^{n}}=$ $\left[\mathbf{v a} \mathbf{a}_{1} \mathbf{v a}_{2} \ldots \mathbf{v a}_{2^{n}}\right]$. We continue this procedure until all the $2^{n}+1$ bases are obtained [12].

For example, we consider the scenario where the transmitter is equipped with $N_{t}^{\mathrm{RF}}=4 \mathrm{RF}$ chains. For $N_{t}^{\mathrm{RF}}=4$, the Hadamard matrix $\mathbf{A}$ is given by:

$$
\mathbf{A}=\frac{1}{2}\left[\begin{array}{cccc}
1 & 1 & 1 & 1 \\
1 & 1 & -1 & -1 \\
1 & -1 & -1 & 1 \\
1 & -1 & 1 & -1
\end{array}\right]
$$

The factor $1 / 2$ is to ensure that (10) satisfies (8). Now the vector $\mathbf{v}_{1}$ is chosen such that the magnitude of all elements of $\mathbf{A}^{H} \mathbf{v}_{i}$ is 1. Solving for the vectors results in three possible vectors, which are:

$$
\mathbf{v}_{1}=\left[\begin{array}{c}
1 \\
-1 \\
-i \\
-i
\end{array}\right], \mathbf{v}_{2}=\left[\begin{array}{c}
1 \\
-i \\
-i \\
-1
\end{array}\right], \mathbf{v}_{3}=\left[\begin{array}{c}
1 \\
i \\
1 \\
-i
\end{array}\right]
$$

Then, the set of MUBs is given by: $\mathbf{B}=\mathbf{v}_{1} \otimes \mathbf{A}, \mathbf{C}=$ $\mathbf{v}_{2} \otimes \mathbf{A}$, and $\mathbf{D}=\mathbf{v}_{3} \otimes \mathbf{A}$.

Given that Hadamard matrix $\mathbf{A}$ in (10) and by applying element-wise operation on $\mathbf{A}$ with vectors $\mathbf{v}_{1}, \mathbf{v}_{2}$, and $\mathbf{v}_{3}$, we arrive at

$$
\begin{aligned}
& \mathbf{B}=\frac{1}{2}\left[\begin{array}{cccc}
1 & 1 & 1 & 1 \\
-1 & -1 & 1 & 1 \\
-i & i & i & -i \\
-i & i & -i & i
\end{array}\right], \mathbf{C}=\frac{1}{2}\left[\begin{array}{cccc}
1 & 1 & 1 & 1 \\
-i & -i & i & i \\
-i & i & i & -i \\
-1 & 1 & -1 & 1
\end{array}\right], \\
& \mathbf{D}=\frac{1}{2}\left[\begin{array}{cccc}
1 & 1 & 1 & 1 \\
i & i & -i & -i \\
1 & -1 & -1 & 1 \\
-i & i & -i & i
\end{array}\right] .
\end{aligned}
$$

Note that the identity matrix is also an MUB, since it satisfies (8). Thus, we have obtained $N_{t}^{\mathrm{RF}}+1=5 \mathrm{MUBs}^{1}$. However, since the identity matrix does not explicitly perform any linear processing of the input streams, it can be discarded in order to reduce the feedback overhead. Nonetheless, to leverage any benefits offered by the identity matrix, the transmitter can always keep a check on it by comparing its performance to that of non-trivial MUBs' performance. The left circular shift of the columns of each matrix $\mathbf{A}, \mathbf{B}, \mathbf{C}$ and $\mathbf{D}$ by $p$ modulo 4 places, where $p=1,2,3,4$, forms the codebook entries, with cardinality $\mathcal{C}=16$, relying on a 4-bit feedback.

Thus, the set of TPC matrices of the codebook $\mathcal{F}$ after employing the left circular shift by $p$ modulo 4 places to each matrix, the codebook can be expressed as $\mathcal{F}=$ $\left\{\mathbf{A}_{0}, \mathbf{A}_{1}, \mathbf{A}_{2}, \mathbf{A}_{3}, \mathbf{B}_{0}, \mathbf{B}_{1}, \mathbf{B}_{2}, \mathbf{B}_{3}, \mathbf{C}_{0}, \mathbf{C}_{1}, \mathbf{C}_{2}, \mathbf{C}_{3}, \mathbf{D}_{0}, \mathbf{D}_{1}\right.$, $\left.\mathbf{D}_{2}, \mathbf{D}_{3}\right\}$.

\footnotetext{
${ }^{1}$ An alternate method for construction of MUBs is presented in [16].
} 
Having obtained the codebook $\mathcal{F}$, we select the first $N_{s}$ columns of the specific TPC matrix from the codebook which maximizes the minimum $\mathrm{SNR}^{2}$ of the effective channel. The $\mathrm{SNR}$ of the $k^{\text {th }}$ stream using a ZF receiver is given by [17]:

$$
\mathrm{SNR}_{k}=\frac{P}{N_{s} N_{o}\left(\mathbf{F}_{\mathrm{BB}}^{*} \mathbf{H}_{\mathrm{eff}}^{*} \mathbf{H}_{\mathrm{eff}} \mathbf{F}_{\mathrm{BB}}\right)_{\mathrm{kk}}^{-1}},
$$

where $P$ and $N_{o}$ are the signal and the noise powers, respectively. Thus, the TPC $\mathbf{F}_{\mathrm{BB}}$ which maximizes the minimum SNR is selected from the codebook $\mathcal{F}$ as follows:

$$
\mathbf{F}_{\mathrm{BB}}=\arg \max _{\mathbf{F}_{\mathrm{BB}} \in \mathcal{F}} \Lambda_{\min }\left\{\mathbf{H}_{\mathrm{eff}} \mathbf{F}_{\mathrm{BB}}\right\},
$$

where $\Lambda_{\text {min }}$ is the minimum singular value of $\mathbf{H}_{\mathrm{eff}} \mathbf{F}_{\mathrm{BB}}$.

Since the mmWave channel of the adjacent antenna elements is correlated due to their $\lambda / 2$ spacing, employing MUB baseband TPC amalgamated with DFT aided RF beamforming significantly reduces the complexity, while having an improved performance in spatially correlated channels.

The pseudo code for the proposed design is shown below.

Algorithm 1 Proposed RF and Baseband Codebook Design

\section{Acquire codebook $\mathcal{F}$ and store in memory}

1: Obtain vectors $\mathbf{v}_{i}$ from Hadamard matrix $\mathbf{A}_{N_{t}^{\mathrm{RF}}}$ such that magnitude of all elements of $\mathbf{A}^{H} \mathbf{v}_{i}=1$;

2: Obtain MUBs by element-wise multiplication of $\mathbf{v}_{i}$ with A, $\forall 1 \leq i \leq N_{t}^{\mathrm{RF}}-1$;

3: Obtain the codebook $\mathcal{F}$ by left circular shift of each MUB to $p$ places, where $p=1, \ldots, N_{t}^{\mathrm{RF}}-1$;

\section{Selection of TPC from the codebook}

1: We have $\mathbf{H}=\mathbf{U} \Sigma \mathbf{V}^{H}$;

2: Obtain columns of $\mathbf{F}_{\mathrm{RF}}$ and $\mathbf{W}_{\mathrm{RF}}$ from $\mathbf{D F} \mathbf{T}_{N_{t}}$ such that $\mathbf{F}_{\mathrm{RF}}=\max <\mathbf{D F T}_{N_{t}}(:, i), \mathbf{V}>, \quad 1 \leq i \leq N_{t}^{\mathrm{RF}}$, and $\mathbf{W}_{\mathrm{RF}}=\max <\mathbf{D F T}_{N_{r}}(:, i), \mathbf{U}>, 1 \leq i \leq N_{r}^{\mathrm{RF}}$;

3: Set the effective channel to $\mathbf{H}_{\mathrm{eff}}=\mathbf{W}_{\mathrm{RF}}^{H} \mathbf{H F}_{\mathrm{RF}}$;

4: Select the TPC from the codebook which satisfies: $\mathbf{F}_{\mathrm{BB}}=$ $\arg \max _{\mathbf{F}_{\mathrm{BB}} \in \mathcal{F}} \Lambda_{\min }\left\{\mathbf{H}_{\mathrm{eff}} \mathbf{F}_{\mathrm{BB}}\right\}$;

5: $\mathbf{W}_{\mathrm{BB}}$ is chosen as $\mathrm{ZF}$ combiner.

\section{Complexity and Properties of the MUB Codebook}

The codebook search complexity is quantified in terms of the number of computations required for finding the best TPC matrix.

To find the best TPC from the codebook we have to perform $\mathcal{C} N_{r}^{\mathrm{RF}}\left(N_{t}^{\mathrm{RF}}-1\right)$ complex-valued additions [13], where $\mathcal{C}$ is the cardinality of the codebook, but no complex multiplications are required. On the other hand, codebooks such as the Grassmannian and Fourier designs would require $\mathcal{C} N_{s}\left(N_{r}^{\mathrm{RF}}\right)^{2}$ complex multiplications plus $\mathcal{C}\left(N_{r}^{\mathrm{RF}}\right)^{2}\left(N_{s}-1\right)$ additions [17], while a typical digital TPC operating in the baseband relying on perfect CSI, such as [18] using the same number of RF chains would need $N_{s} N_{r}^{\mathrm{RF}}\left(N_{t}^{\mathrm{RF}}-1\right)$ complex-valued additions and $N_{s} N_{r}^{\mathrm{RF}} N_{t}^{\mathrm{RF}}$ multiplications. Thus, the MUB based codebook significantly reduces the overall search complexity.

Furthermore, the entries of the codebook are constructed from a finite alphabet $\mathcal{X}=\{i,-i, 1,-1\}$, which meets the

\footnotetext{
${ }^{2}$ Minimum SNR of all links between each Tx and Rx antenna.
}

constant modulus constraint, and hence avoids any potential power imbalance for the system. Additionally, the codebook exhibits a nested property, which means that the computations that are performed for higher-rank transmission can be reused for lower-rank transmission. In other words, the rank 1 TPC is a subset of the rank 2 TPC, the rank 2 TPC is a subset of the rank 3 and the rank 3 TPC is a subset of the rank 4 TPC [12] as illustrated below.

$$
\text { Rank } 1 \subset \text { Rank } 2 \subset \text { Rank } 3 \subset \text { Rank } 4
$$$$
\text { IV. Simulation Results }
$$

In this section, we present our simulation results for characterizing both the performance and the achievable rate when the DFT-MUB based RF and baseband precoding is employed. We performed Monte Carlo simulations for performance comparison between the baseband TPC based on SVD of the channel and the hybrid precoding relying on limited-feedback. The system configuration and parameters, such as the number of RF chains, antennas, and modulation schemes are shown in Table I.

TABLE I: Simulation paramete
\begin{tabular}{|l|l|}
\hline Parameters & Values \\
\hline \hline Modulation & 4 QAM \\
\hline$N_{c}$ & 4 \\
\hline$N_{\text {ray }}$ & 6 \\
\hline$N_{t}$ & 32,8 \\
\hline$N_{r}$ & 16,8 \\
\hline$N_{s}$ & 2 \\
\hline$N_{t}^{\mathrm{RF}}$ & 4 \\
\hline$N_{r}^{\mathrm{RF}}$ & 4 \\
\hline$\phi_{n_{c}}^{n_{r a y}}$ & $\sim \mathcal{U}[0,2 \pi)$ \\
\hline
\end{tabular}

Fig. 2 plots the achievable rates of digital precoding using SVD, of OMP [18], of DFT-MUB, and DFT-Identity, where the identity matrix is chosen as the baseband TPC, using (5) for a $8 \times 8$ and $32 \times 16$ MIMO sytem. In this figure, the DFT-MUB based codebook design using 4-bit feedback is investigated. In this configuration, two spatial streams were transmitted using $4 \mathrm{RF}$ chains both at the transmitter and at the receiver. It is seen from Fig. 2 that the performance gap between the SVD based TPC and the DFT-MUB aided codebook is around $2.5 \mathrm{~dB}$ for the $32 \times 16$ and as low as $0.8 \mathrm{~dB}$ for the $8 \times 8 \mathrm{MIMO}$ configurations considered. Furthermore, there is a gap of around $1 \mathrm{~dB}$ against the OMP aided precoding design for a $32 \times 16$ MIMO, whereas for an $8 \times 8$ MIMO the performance of the DFT-MUB overlaps with the OMP. We also compared our results against the DFT-Identity based system, where the TPC is constructed by selecting first $N_{s}$ columns of the identity matrix. Against the DFT-Identity aided system, there is an approximately $0.5 \mathrm{~dB}$ gain when DFT-MUB is performed.

Additionally, it is important to quantify the complexity of different designs considered. For $N_{t}^{\mathrm{RF}}=4$ and $N_{r}^{\mathrm{RF}}=4$, the MUB based TPC would need only 192 complex additions. On the other hand, the Grassmannian and Fourier codebooks based TPC would need 512 complex additions plus 256 complex multiplications, as described in Sec. III-C. Although the TPC relying on perfect CSI such as OMP [18] would require 24 


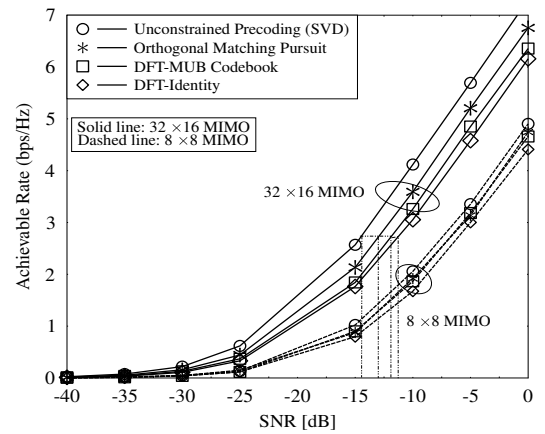

Fig. 2: Comparison of achievable rate using DFT-MUB based codebook design with 4-bit feedback and different other methods relying on perfect CSI for $32 \times 16$ and $8 \times 8$ MIMO configurations. In this configuration, we have $N_{s}=2$ and $N_{t}^{\mathrm{RF}}=N_{r}^{\mathrm{RF}}=4$.

complex additions plus 32 complex multiplications, it imposes a high overhead as it depends on perfect CSI. Furthermore, the SVD based TPC relies completely on digital processing elements, where DACs and automatic gain controls (AGCs) are needed for each antenna element, which imposes a high power consumption and high hardware complexity.

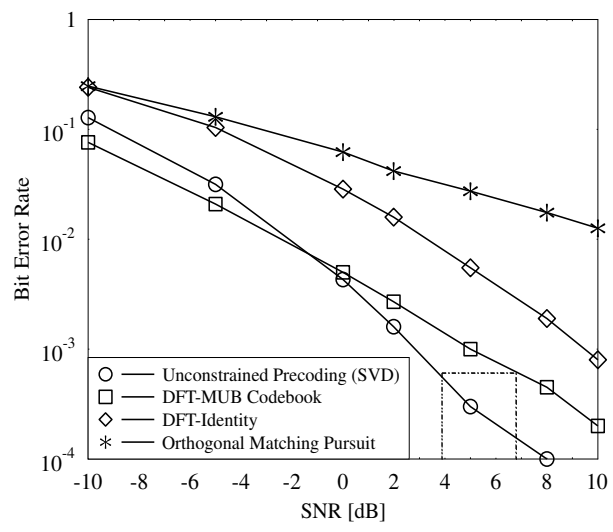

Fig. 3: Comparison of uncoded bit error rate (BER) of a $8 \times 8$ MIMO between on DFT-MUB based codebook design with 4-bit feedback and different other methods relying on perfect CSI. In this configuration, we have $N_{s}=2$ and $N_{t}^{\mathrm{RF}}=N_{r}^{\mathrm{RF}}=$ 4.

We also performed Monte Carlo simulations to investigate the attainable uncoded bit error rate (BER) performance for an $8 \times 8$ MIMO system using 4 QAM. In this configuration, two spatial streams using $4 \mathrm{QAM}$ were transmitted over $N_{t}^{\mathrm{RF}}=4$ and $N_{r}^{\mathrm{RF}}=4 \mathrm{RF}$ chains. Fig. 3 shows the comparison between the BERs of the DFT-MUB aided codebook design using 4-bit feedback, of the SVD based TPC, of the DFT-Identity and of OMP. It is seen that at low SNRs the DFT-MUB codebook design outperforms the SVD based TPC. It is instructive to note that the SVD based TPC is sub-optimal in terms of BER. Furthermore, at the BER of $10^{-3}$ the performance gap between the DFT-MUB based design and the SVD design is around $2.5 \mathrm{~dB}$, despite the DFT-MUB scheme's constant modulus nature and significantly lower complexity. DFT-MUB also outperforms the DFT-Identity and OMP designs.

\section{CONCLusions}

In this paper, we investigated a hybrid codebook design based on the combination of the DFT in the RF and MUB in the baseband for mmWave MIMO systems. We demonstrated that the proposed design performs close to the optimal unconstrained precoder (SVD) in terms of achievable rate. Furthermore, our design has lower BER than that of OMP. Additionally, our system performs within $2.5 \mathrm{~dB}$ from the SVD based precoding with significantly lower complexity than orthogonal matching pursuit and SVD.

\section{REFERENCES}

[1] A. Ghosh, R. Ratasuk, B. Mondal, N. Mangalvedhe, and T. Thomas, "Lte-advanced: next-generation wireless broadband technology," IEEE Wireless Communications, vol. 17, no. 3, pp. 10-22, June 2010.

[2] Y. Niu et al., "A survey of millimeter wave (mmwave) communications for 5G: Opportunities and challenges," CoRR, vol. abs/1502.07228, 2015.

[3] G. Maccartney, M. Samimi, and T. Rappaport, "Exploiting directionality for millimeter-wave wireless system improvement," in IEEE International Conference on Communications (ICC), June 2015, pp. 2416-2422.

[4] B. Le et al., "Analog-to-digital converters," IEEE Signal Processing Magazine, vol. 22, no. 6, pp. 69-77, 2005.

[5] X. Zhang, A. F. Molisch, and S.-Y. Kung, "Variable-phase-shift-based RF-baseband codesign for MIMO antenna selection," IEEE Transactions on Signal Processing, vol. 53, no. 11, pp. 4091-4103, 2005.

[6] S. Han et al., "Large-scale antenna systems with hybrid analog and digital beamforming for millimeter wave 5G," IEEE Communications Magazine, vol. 53, no. 11, pp. 186-194, 2015.

[7] J. Song, J. Choi, and D. J. Love, "Codebook design for hybrid beamforming in millimeter wave systems," in IEEE International Conference on Communications, (ICC), June 2015.

[8] R. Rajashekar and L. Hanzo, "Hybrid beamforming in mm-wave MIMO systems having a finite input alphabet," IEEE Transactions on Communications, vol. 64, no. 8, pp. 3337 - 3349, 2016.

[9] A. Alkhateeb and R. W. Heath, "Frequency selective hybrid precoding for limited feedback millimeter wave systems," IEEE Transactions on Communications, vol. 64, no. 5, pp. 1801 - 1818, April 2016.

[10] M. E. Eltayeb, A. Alkhateeb, R. W. Heath, and T. Y. Al-Naffouri, "Opportunistic beam training with hybrid analog/digital codebooks for mmwave systems," in IEEE Global Conference on Signal and Information Processing (GlobalSIP), Dec. 2015.

[11] B. Clerckx, Y. Zhou, and S. Kim, "Practical codebook design for limited feedback spatial multiplexing," in IEEE International Conference on Communications, 2008, pp. 3982-3987.

[12] B. Mondal, T. A. Thomas, and M. Harrison, "Rank-independent codebook design from a quaternary alphabet," in Conference Record of the Forty-First Asilomar Conference on Signals, Systems and Computers, 2007, pp. $297-301$.

[13] T. Inoue and R. Heath, "Kerdock codes for limited feedback precoded MIMO systems," IEEE Transactions on Signal Processing, vol. 57, no. 9, pp. 3711 - 3716, 2009.

[14] D. Yang, L.-L. Yang, and L. Hanzo, "DFT-based beamforming weightvector codebook design for spatially correlated channels in the unitary precoding aided multiuser downlink," in IEEE International Conference on Communications (ICC), May 2010.

[15] M. K. Samimi and T. S. Rappaport, "3-D millimeter-wave statistical channel model for 5G wireless system design," IEEE Transactions on Microwave Theory and Techniques, vol. 64, no. 7, pp. 2207-2225, July 2016.

[16] A. Klappenecker and M. Rotteler, "Constructions of mutually unbiased bases," CoRR, vol. quant-ph/0309120v1, 2003. [Online]. Available: http://arxiv.org/abs/quant-ph/0309120v1

[17] A. Alkhateeb, G. Leus, and J. Robert W. Heath, "Limited feedback hybrid precoding for multi-user millimeter wave systems," IEEE Transactions on Wireless Communications, vol. 14, no. 11, pp. 3337 - 3349, 2015.

[18] O. El Ayach, S. Rajagopal, S. Abu-Surra, Z. Pi, and R. Heath, "Spatially sparse precoding in millimeter wave MIMO systems," IEEE Transactions on Wireless Communications, vol. 13, no. 3, pp. 1499-1513, March 2014. 